\title{
The Theory of Materials Failure
}

\author{
Richard M. Christensen
}

Stanford University

\section{OXFORD}




\section{Contents}

Recognition xi

Technical Status and Challenges xii

1 The Perspective on Failure and Direction of Approach 1

1.1 Materials Failure Problem 1

1.2 Direction and Scope $\quad 2$

1.3 Guides for Utility 4

References 5

2 History, Conditions, and Requirements 6

2.1 Historical Review $\quad 7$

2.2 Conditions and Requirements of Study 12

$\begin{array}{ll}\text { References } & 14\end{array}$

3 Isotropic Baselines $\quad 16$

3.1 Failure Characterization $\quad 16$

$\begin{array}{lll}3.2 & \text { Stress versus Strain } & 17\end{array}$

3.3 Mises and Tresca Failure Criteria $\quad 19$

3.4 Drucker-Prager Failure Criterion $\quad 23$

3.5 Coulomb-Mohr Failure Criterion $\quad 25$

3.6 The Bottom Line 28

$\begin{array}{ll}\text { References } & 29\end{array}$

4 The Failure Theory for Isotropic Materials $\quad 30$

4.1 Theoretical and Testing Problems 30

4.2 Properties or Parameters 31

4.3 The Organizing Principle 32

4.4 The Constitutive Equations of Failure, Part A:
Polynomial-Invariants Criterion

4.5 The Constitutive Equations of Failure, Part B:

4.6 Ductile and Brittle Limits $\quad 42$

4.7 Problem Sets Purpose $\quad 46$

Problem Areas for Study $\quad 48$

$\begin{array}{ll}\text { References } & 49\end{array}$ 
viii Contents

5 Isotropic Materials Failure Behavior $\quad \mathbf{5 0}$

5.1 Failure Behavior in Two Dimensions 51

5.2 Failure in Principal Stress Space $\quad 51$

$\begin{array}{lll}5.3 & \text { Ductile-versus-Brittle Failure } & 57\end{array}$

$5.4 T$ and $C$ versus $S$ and $D \quad 61$

Problem Areas for Study $\quad 68$

$\begin{array}{ll}\text { References } & 69\end{array}$

6 Experimental and Theoretical Evaluation $\quad 70$

$\begin{array}{ll}6.1 \text { Evaluation Problem } & 70\end{array}$

6.2 Theoretical Assessment $\quad 72$

$\begin{array}{lll}6.3 & \text { Experimental Evaluation } & 76\end{array}$

6.4 Isotropy Conclusion $\quad 83$

Problem Areas for Study $\quad 84$

References $\quad 85$

7 Failure Theory Applications $\quad \mathbf{8 7}$

7.1 Very Ductile Polymers $\quad 88$

$\begin{array}{lll}7.2 & \text { Brittle Polymers } & 89\end{array}$

$\begin{array}{lll}7.3 \text { Glasses } & 91\end{array}$

$\begin{array}{lll}7.4 \text { Ceramics } & 92\end{array}$

$\begin{array}{ll}7.5 \text { Minerals } & 93\end{array}$

$\begin{array}{lll}7.6 \text { Geo-Materials } & 95\end{array}$

Problem Areas for Study $\quad 97$

$\begin{array}{ll}\text { References } & 97\end{array}$

8 The Ductile/Brittle Transition for Isotropic

8.1 Introduction $\quad 98$

8.2 Conventional Difficulty with Characterizing Ductility 100

8.3 The Ductile/Brittle Transition 103

8.4 The Failure Number for Gauging Ductility Levels 108

Problem Areas for Study $\quad 115$

$\begin{array}{ll}\text { References } & 117\end{array}$

9 Defining Yield Stress and Failure Stress (Strength) 118

9.1 Yield Stress and Strength as Historically and Currently Practiced 118

$\begin{array}{lll}9.2 & \text { A Rational Definition of Yield Stress } & 119\end{array}$

9.3 A Rational Definition of Failure Stress 124 
Contents ix

9.4 Significance and Conclusions 130

Problem Areas for Study $\quad 131$

References 132

10 Fracture Mechanics $\quad 133$

10.1 Fracture Mechanics Development 133

10.2 The Two Distinct Failure Theories 136

10.3 Fracture Mechanics Example $\quad 137$

10.4 Failure Criterion Example $\quad 139$

$\begin{array}{ll}10.5 \text { Assessment } & 141\end{array}$

Problem Areas for Study $\quad 142$

$\begin{array}{ll}\text { References } & 143\end{array}$

11 Anisotropic Unidirectional Fiber
Composites Failure

11.1 Transversely Isotropic Polynomial Invariants 144

11.2 The Matrix-Controlled Failure Criterion $\quad 146$

11.3 The Fiber-Controlled Failure Criterion $\quad 147$

$\begin{array}{lr}11.4 \text { Hashin Failure Criterion } & 149\end{array}$

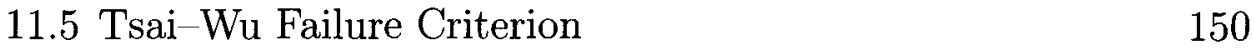

11.6 Comparisons 151

Problem Areas for Study $\quad 154$

$\begin{array}{ll}\text { References } & 155\end{array}$

12 Anisotropic Fiber Composite Laminates Failure $\quad 157$

$\begin{array}{ll}12.1 \text { Introduction } & 157\end{array}$

12.2 Progressive Damage in Laminates 161

12.3 Testing Results 166

12.4 Polynomial Invariants for Laminates 168

Problem Areas for Study $\quad 175$

$\begin{array}{ll}\text { References } & 175\end{array}$

13 Micromechanics Failure Analysis $\quad 177$

$\begin{array}{ll}13.1 \text { General Considerations } & 177\end{array}$

13.2 Transverse Shear Strength for Aligned
Fiber Composites

13.3 Spherical Inclusion in an Infinite Elastic Medium 187

13.4 Load Redistribution in Aligned Fiber Composites $\quad 192$

Problem Areas for Study 198

$\begin{array}{ll}\text { References } & 199\end{array}$ 
$\times$ Contents

14 Nanomechanics Failure Analysis 200

14.1 Graphene Nanostructure 200

14.2 A Hypothetical Nanostructure 206

14.3 Comparison and Discussion 207

14.4 Are the Elements Ductile or Brittle? 210

14.5 A Ductility Scale for the Elements 219

Problem Areas for Study 222

References 222

15 Damage, Cumulative Damage, Creep
and Fatigue Failure

15.1 Damage 223

15.2 Cumulative Damage 226

15.3 Four Models 229

15.4 Residual Strength 236

15.5 Life Prediction 238

15.6 Residual Life 241

15.7 Conclusion $\quad 242$

Problem Areas for Study $\quad 243$

References 244

16 Probabilistic Failure and Probabilistic

Life Prediction $\quad 245$

16.1 Variability and Extreme Cases of Variability 245

16.2 Power-Law Failure Interpretation 247

16.3 Weibull Distribution Physical Basis 250

16.4 Kinetic Crack Theory and Life Prediction 255

16.5 Probabilistic Generalization of Strength and
Lifetime Theory

Problem Areas for Study $\quad 269$

References 269

Index $\quad 273$ 